\title{
A Hybrid Intelligent Algorithm for Optimal Birandom Portfolio Selection Problems
}

\author{
Qi Li, Guo-Hua Cao, and Dan Shan \\ School of Economics and Business Administration, Chongqing University, Chongqing 400030, China \\ Correspondence should be addressed to Guo-Hua Cao; caoguohua@cqu.edu.cn
}

Received 4 May 2014; Accepted 31 May 2014; Published 16 June 2014

Academic Editor: He Huang

Copyright (C) 2014 Qi Li et al. This is an open access article distributed under the Creative Commons Attribution License, which permits unrestricted use, distribution, and reproduction in any medium, provided the original work is properly cited.

Birandom portfolio selection problems have been well developed and widely applied in recent years. To solve these problems better, this paper designs a new hybrid intelligent algorithm which combines the improved LGMS-FOA algorithm with birandom simulation. Since all the existing algorithms solving these problems are based on genetic algorithm and birandom simulation, some comparisons between the new hybrid intelligent algorithm and the existing algorithms are given in terms of numerical experiments, which demonstrate that the new hybrid intelligent algorithm is more effective and precise when the numbers of the objective function computations are the same.

\section{Introduction}

Portfolio theory was initially put forward by Markowitz [1] and has received great development since then. The main content of it is to study how to allocate one's capital to a large number of securities and the study mainly focuses on three aspects: the first is how to estimate the security return; the second is how to build portfolio models; the third is how to design efficient algorithms to solve these models. Many scholars have made great contributions in this field.

In the early literatures, security return was assumed to obey normal distribution. However, the following researches manifested that the assumption of normal distribution did not accord with the facts. Therefore, a lot of new distributions were used to describe the security return [2-9]. Among them, birandom distribution received great attention and development by some scholars [7-9] and literature [9] demonstrated that birandom distribution could reflect the features of securities' technical patterns and the investors' heterogeneity.

When the security return followed birandom distribution, the existing literatures established several birandom portfolio models and designed the corresponding hybrid intelligent algorithms [7-9]. However, all these algorithms were based on genetic algorithm (GA) and existed some common shortcomings, such as low accuracy and inferior local search ability. To solve these birandom portfolio models effectively, this paper designs a new hybrid intelligent algorithm which integrates the improved LGMS-FOA algorithm and birandom simulation. The experimental results show that the new algorithm is more efficient.

The rest of this paper is organized as follows: Section 2 recalls some basic concepts about birandom theory; Section 3 provides an overview of birandom portfolio models; Section 4 presents a new hybrid intelligent algorithm which integrates the improved LGMS-FOA algorithm and birandom simulation; Section 5 provides numerical examples to test the effectiveness of the new hybrid intelligent algorithm; finally, a brief summary about this paper is given.

\section{Birandom Theory}

Definition 1. A birandom variable $\varepsilon$ is a mapping from a probability space $(\Omega, A, \operatorname{Pr})$ to a collection of random variables such that, for any Borel subset $B$ of the real line $R$, the induced function $\operatorname{Pr}(\varepsilon(w) \in B)$ is a measurable function with respect to $w[7]$.

Example 2. Let $\Omega=\left(\omega_{1}, \omega_{2}\right)$ ( $\omega_{1}$ represents security rising and $\omega_{2}$ represents security falling) and $\operatorname{Pr}\left(\omega_{1}\right)=a$ and 
$\operatorname{Pr}\left(\omega_{2}\right)=b$. Assume that $\varepsilon$ is a function on $(\Omega, A, \operatorname{Pr})$ as follows:

$$
\varepsilon(\omega)= \begin{cases}\varepsilon_{1} & \text { if } \omega=\omega_{1} \\ \varepsilon_{2} & \text { if } \omega=\omega_{2}\end{cases}
$$

where $\varepsilon_{1}$ and $\varepsilon_{2}$ follow uniform distribution; then $\varepsilon$ is a birandom variable according to Definition 1 .

Definition 3. Let $\varepsilon=\left(\varepsilon_{1}, \varepsilon_{2}, \ldots, \varepsilon_{n}\right)$ be a birandom vector on $(\Omega, A, \operatorname{Pr})$, and let $f: R^{n} \rightarrow R^{m}$ be a vector-valued Borel measurable function. Then the primitive chance of birandom event characterized by $f(\varepsilon) \leq 0$ is a function from $(0,1]$ to $[0,1]$, defined as $[8]$

$$
\begin{aligned}
\operatorname{Ch} & \{f(\varepsilon) \leq 0\}(\alpha) \\
& =\sup \{\beta \mid \operatorname{Pr}\{\omega \in \Omega \mid \operatorname{Pr}\{f(\varepsilon(\omega)) \leq 0\} \geq \beta\} \geq \alpha\} .
\end{aligned}
$$

Definition 4. Let $\varepsilon$ be a birandom variable defined on the probability space $(\Omega, A, \operatorname{Pr})$. Then the expected value of birandom variable $\varepsilon$ is defined as

$$
\begin{aligned}
E(\varepsilon)= & \int_{0}^{\infty} \operatorname{Pr}\{\omega \in \Omega \mid E[\varepsilon(\omega)] \geq t\} d t \\
& -\int_{-\infty}^{0} \operatorname{Pr}\{\omega \in \Omega \mid E[\varepsilon(\omega)] \leq t\} d t,
\end{aligned}
$$

provided that at least one of the above two integrals is finite [8].

\section{Birandom Portfolio Models}

In this section, we provide an overview of birandom portfolio models. Let $x_{i}$ represent the investment proportion in security $i$ and let $k_{i}$ denote the return of the $i$ th security for $i=1,2, \ldots, n$, respectively. In particular, $k_{i}$ follows birandom distribution for $i=1,2, \ldots, n$. Depending on different measures of investment profit and risk, birandom portfolio models are divided into different types.

3.1. Birandom Safety-First Model. For each portfolio $x=$ $\left(x_{1}, x_{2}, \ldots, x_{n}\right)$, the chance that return of total asset (RTA) is no less than a disaster level is used to represent investment risk, which is given by

$$
\operatorname{Ch}\left\{x_{1} k_{1}+x_{2} k_{2}+\cdots+x_{n} k_{n} \geq m\right\}\left(\beta_{1}\right) \geq a \text {. }
$$

The chance that RTA is no less than some value is regarded to measure investment profit, which is represented by

$$
\operatorname{Ch}\left\{x_{1} k_{1}+x_{2} k_{2}+\cdots+x_{n} k_{n} \geq t\right\}\left(\beta_{2}\right) \text {, }
$$

where $m$ and $t$ represent the disaster level and profit, respectively. $\beta_{1}, \beta_{2}$, and $a$ represent the corresponding confidence level.
Using (4) and (5), we obtained the following Birandom safety-first model, which was proposed in [9]:

$$
\begin{array}{ll}
\max & \operatorname{Ch}\left\{x_{1} k_{1}+x_{2} k_{2}+\cdots+x_{n} k_{n} \geq t\right\}\left(\beta_{2}\right) \\
\text { s.t. } & \operatorname{Ch}\left\{x_{1} k_{1}+x_{2} k_{2}+\cdots+x_{n} k_{n} \geq m\right\}\left(\beta_{1}\right) \geq a \\
& \sum_{i=1}^{n} x_{i}=1 \\
& x_{i} \geq 0 \quad i=1,2, \ldots, n .
\end{array}
$$

3.2. Birandom Expected Value Model. In this model, investment risk was represented by (4) and the expect value of RTA was used to measure investment profit which was given by

$$
E\left[x_{1} k_{1}+x_{2} k_{2}+\cdots+x_{n} k_{n}\right]
$$

Based on (4) and (7), Birandom expect value model was proposed in [8], which was formulated as follows:

$$
\begin{array}{ll}
\max & E\left[x_{1} k_{1}+x_{2} k_{2}+\cdots+x_{n} k_{n}\right] \\
\text { s.t. } & \operatorname{Ch}\left\{x_{1} k_{1}+x_{2} k_{2}+\cdots+x_{n} k_{n} \geq m\right\}\left(\beta_{1}\right) \geq a \\
& \sum_{i=1}^{n} x_{i}=1 \\
& x_{i} \geq 0 \quad i=1,2, \ldots, n .
\end{array}
$$

3.3. Birandom Chance-Constrained Model. Literature [7] proposed birandom chance-constrained model, which was listed as below:

$$
\begin{array}{ll}
\max & \operatorname{Ch}\left\{x_{1} k_{1}+x_{2} k_{2}+\cdots+x_{n} k_{n} \geq t\right\}\left(\beta_{2}\right) \\
\text { s.t. } & \sum_{i=1}^{n} x_{i}=1 \\
& x_{i} \geq 0 \quad i=1,2, \ldots, n .
\end{array}
$$

Remark 5. The parameters of models (6), (8), and (9) have the same economic meaning.

Remark 6. For more details on these models, please refer to the corresponding literatures.

\section{Hybrid Intelligent Algorithm}

In this section, a new hybrid intelligent algorithm is designed to solve models (6), (8), and (9), where the improved LGMSFOA algorithm and birandom simulation are used.

4.1. Birandom Simulation. Because of the uncertainty of birandom variable, we should use birandom simulation [10] to solve equations (4), (5), and (7). 
Algorithm 1 (birandom simulation for (4)).

Step 1. Set $l=1$.

Step 2. Generate $w_{1}, w_{2}, \ldots, w_{N}$ from $\Omega$ according to the probability Pr. Consider

$$
w_{i}=\left\{y_{1}, y_{2}, \ldots, y_{n}\right\}, \quad i=1,2, \ldots, N .
$$

Step 3. Compute the probability

$$
\begin{array}{r}
\delta_{i}=\operatorname{Pr}\left\{x_{1} k_{1}\left(y_{1}\right)+x_{2} k_{2}\left(y_{2}\right)+\cdots+x_{n} k_{n}\left(y_{n}\right) \geq m\right\}, \\
i=1,2, \ldots, N,
\end{array}
$$

by stochastic simulation.

Step 4. Set $N^{*}$ as the integer part of $\beta_{1} N$.

Step 5. Return the $N^{*}$ th largest element $\delta^{*}$ in $\left\{\delta_{1}, \delta_{2}, \ldots, \delta_{N}\right\}$.

Step 6. If $\delta^{*} \geq a$, then $l=l * 1$; else $l=l * 0$.

Step $7 . l=1$ means that the solution is feasible; $l=0$ means that the solution is infeasible.

Algorithm 2 (birandom simulation for (5)).

Step 1. Generate $w_{1}, w_{2}, \ldots, w_{N}$ from $\Omega$ according to the probability Pr. Consider

$$
w_{i}=\left\{y_{1}, y_{2}, \ldots, y_{n}\right\}, \quad i=1,2, \ldots, N .
$$

Step 2. Compute the probability

$$
\begin{array}{r}
\delta_{i}=\operatorname{Pr}\left\{x_{1} k_{1}\left(y_{1}\right)+x_{2} k_{2}\left(y_{2}\right)+\cdots+x_{n} k_{n}\left(y_{n}\right) \geq t\right\}, \\
i=1,2, \ldots, N,
\end{array}
$$

by stochastic simulation.

Step 3. Set $N^{*}$ as the integer part of $\beta_{2} N$.

Step 4. Return the $N^{*}$ th largest element $\delta^{*}$ in $\left\{\delta_{1}, \delta_{2}, \ldots, \delta_{N}\right\}$. Algorithm 3 (birandom simulation for (7)).

Step 1. Set $e=0$.

Step 2. Generate $w_{1}, w_{2}, \ldots, w_{N}$ from $\Omega$ according to the probability Pr. Consider

$$
w_{i}=\left\{y_{1}, y_{2}, \ldots, y_{n}\right\}, \quad i=1,2, \ldots, N .
$$

Step 3. Compute the probability

$$
\begin{array}{r}
\delta_{i}=E\left[x_{1} k_{1}\left(y_{1}\right)+x_{2} k_{2}\left(y_{2}\right)+\cdots+x_{n} k_{n}\left(y_{n}\right)\right], \\
i=1,2, \ldots, N,
\end{array}
$$

by stochastic simulation.

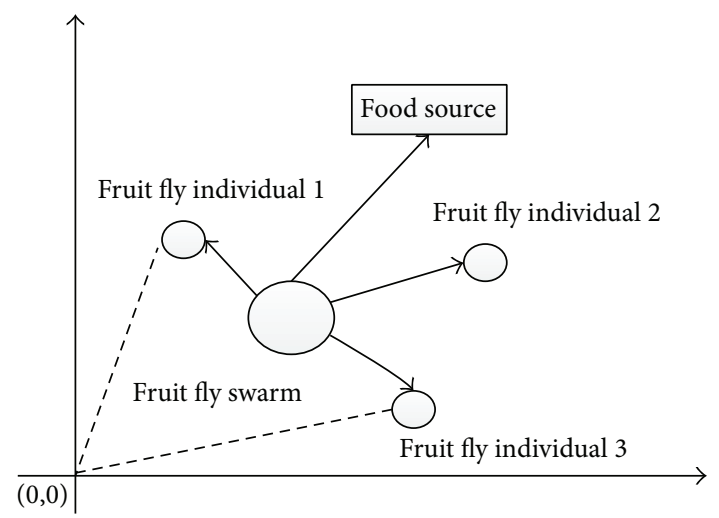

FIGURE 1: Food finding process of fruit fly swarm.
Step 4. Consider

$$
e \longleftarrow e+E\left[x_{1} k_{1}\left(y_{1}\right)+x_{2} k_{2}\left(y_{2}\right)+\cdots+x_{n} k_{n}\left(y_{n}\right)\right]
$$

Step 5. Repeat the second to the fourth steps for $N$ times.

Step 6. Consider

$$
E\left[x_{1} k_{1}+x_{2} k_{2}+\cdots+x_{n} k_{n}\right]=\frac{e}{N} .
$$

4.2. The Improved LGMS-FOA. Recently, optimization algorithms are widely used in many fields [11-14]. Fruit fly optimization algorithm (FOA) is a new stochastic optimization technique proposed in 2012 [11] and has received rapid developments in recent years. The principle of FOA is based on the food finding behavior of fruit fly swarm. The food finding process was shown in Figure 1 and has two steps: first, it smells food and flies to that direction; then, it uses vision to find food.

To enhance the search ability of FOA, LGMS-FOA was proposed and had been proved to be an effective algorithm by numerical testing [12]. However, LMGS-FOA in the literature [12] is only applied to solve unconstrained optimization problems. To solve birandom portfolio models, the improved LGMS-FOA is proposed by means of combination of LGMSFOA with penalty function method. Without loss of generality, we take model (6), for example, to introduce the improved LGMS-FOA. The steps of it are listed as follows.

Algorithm 4 (the improved LGMS-FOA).

Step 1. Parameters initialization.

The parameters include the number of iteration (maxgen), the population number (sizepop), the searching scope $(r)$, the searching coefficient $(d)$, the primary weight $\left(w_{0}\right)$, and the weight adjustment coefficient $(\rho)$.

Step 2. Initial fruit fly swarm location. 
The fruit fly swarm location $x=\left(x_{1}, x_{2}, \ldots, x_{n}\right)$ is randomly generated in the interval $[-r, r]$ and the searching scope becomes bigger with the increase of $r$.

Step 3. Give the random direction and distance for food finding of an individual fruit fly. Consider

$$
\begin{gathered}
x_{P}=x+w \times d \times(2 \times \operatorname{rand}(1, n)-1), \\
P=1,2, \ldots, \text { sizepop; } \\
w=w_{0} \times \rho^{\text {gen }} .
\end{gathered}
$$

Step 4. Generate fruit fly swarm that fits the constraint $\sum_{i=1}^{n} y_{i}=1$

Step 4.1. A solution $y_{p}=\left(y_{1}, y_{2}, \ldots, y_{n}\right)$ is represented by the individual fruit fly $x_{p}=\left(x_{1}, x_{2}, \ldots, x_{n}\right)$, where the relation between $y_{p}$ and $x_{p}$ is formulated as follows:

$$
y_{i}=\frac{x_{i}}{x_{1}+x_{2}+\cdots+x_{n}}, \quad i=1,2, \ldots, n,
$$

which ensures that $\sum_{i=1}^{n} y_{i}=1$.

Step 4.2. If the element of $y_{p}$ is less than zero, repeat Step 4.1 until the number of individuals is sizepop.

Step 5. Calculate the smell concentration by inputting $y_{p}$ into objective function.

Step 5.1. If $y_{p}=\left(y_{1}, y_{2}, \ldots, y_{n}\right)$ satisfies

$$
\mathrm{Ch}\left\{y_{1} k_{1}+y_{2} k_{2}+\cdots+y_{n} k_{n} \geq m\right\}\left(\beta_{1}\right) \geq a,
$$

then let

$$
\operatorname{Smell}_{i}=\mathrm{Ch}\left\{y_{1} k_{1}+y_{2} k_{2}+\cdots+y_{n} k_{n} \geq t\right\}\left(\beta_{2}\right) .
$$

Step 5.2. If $y_{p}=\left(y_{1}, y_{2}, \ldots, y_{n}\right)$ does not satisfy

$$
\operatorname{Ch}\left\{y_{1} k_{1}+y_{2} k_{2}+\cdots+y_{n} k_{n} \geq m\right\}\left(\beta_{1}\right) \geq a,
$$

then use penalty function method [13] and let

$$
\text { Smell }_{i}=\operatorname{Ch}\left\{y_{1} k_{1}+y_{2} k_{2}+\cdots+y_{n} k_{n} \geq t\right\}\left(\beta_{2}\right)-b,
$$

where $b$ is a given positive integer.

Step 6. Find out the optimal individual among fruit fly swarm. Consider

$$
\text { [best smell best index }]=\max (\text { Smell }) \text {. }
$$

Step 7. Keep the optimal objective function value and the corresponding $x$ coordinate; then the fruit fly swarm flies towards that location:

$$
\begin{aligned}
& \text { Smell best }=\text { best smell } \\
& x=x \text { (best inedx). }
\end{aligned}
$$

Step 8. Repeat Step 2 to Step 7 until termination condition is satisfied.

4.3. Hybrid Intelligent Algorithm. Through integrating the improved LGMS-FOA algorithm and birandom simulation, a new hybrid intelligent algorithm is built and the steps are listed as below.

Algorithm 5 (hybrid intelligent algorithm).

Step 1. Initialize $N$ fruit fly individuals that satisfy the constraints.

Step 2. Calculate the objective function values for all fruit fly individuals by birandom simulation.

Step 3. Find out the optimal objective value.

Step 4. Keep the optimal objective value and the corresponding location of fruit fly individual.

Step 5. Repeat Step 2 to Step 5, until termination condition is satisfied.

\section{Numerical Experiments}

To test the effectiveness of the new hybrid intelligent algorithm, we compared it with the existing algorithm [7-9] through numerical examples.

5.1. Experimental Setup. Assume that the investor selects three securities represented by $k_{1}, k_{2}$, and $k_{3}$. The security return follows the birandom distribution in Example 2 and the corresponding parameters of each security are shown in Table 1. Besides, the investor takes $t=0.04, m=-0.06$, $\beta_{1}=0.7, \beta_{2}=0.5$, and $a=0.6$; then, models (6), (8), and (9) satisfying the above parameters are changed into

$$
\begin{array}{ll}
\max & \operatorname{Ch}\left\{x_{1} k_{1}+x_{2} k_{2}+x_{3} k_{3} \geq 0.04\right\}(0.5) \\
\text { s.t. } & \operatorname{Ch}\left\{x_{1} k_{1}+x_{2} k_{2}+x_{3} k_{3} \geq-0.06\right\}(0.7) \geq 0.6 \\
& x_{1}+x_{2}+x_{3}=1 \\
& x_{i} \geq 0 \quad i=1,2,3, \\
\max \quad & E\left[x_{1} k_{1}+x_{2} k_{2}+x_{3} k_{3}\right] \\
\text { s.t. } \quad & \operatorname{Ch}\left\{x_{1} k_{1}+x_{2} k_{2}+x_{3} k_{3} \geq-0.06\right\}(0.7) \geq 0.6 \\
& \sum_{i=1}^{3} x_{i}=1 \\
& x_{i} \geq 0 \quad i=1,2,3,
\end{array}
$$


TABLE 1: The distributions of securities $k_{1}, k_{2}$, and $k_{3}$.

\begin{tabular}{lccc}
\hline Parameters & $k_{1}$ & $k_{2}$ & $k_{3}$ \\
\hline$a_{i}(i=1,2,3)$ & $3 / 4$ & $1 / 2$ & $1 / 3$ \\
$b_{i}(i=1,2,3)$ & $1 / 4$ & $1 / 2$ & $2 / 3$ \\
$\varepsilon_{i 1}(i=1,2,3)$ & $U[0,7 \%]$ & $U[0,9 \%]$ & $U[0,11 \%]$ \\
$\varepsilon_{i 2}(i=1,2,3)$ & $U[-9 \%, 0]$ & $U[-11 \%, 0]$ & $U[-13 \%, 0]$ \\
\hline
\end{tabular}

TABLE 2: The optimal results of model (26).

\begin{tabular}{lccccc}
\hline \multirow{2}{*}{ Model } & \multirow{2}{*}{ Algorithm } & \multicolumn{3}{c}{ Optimal solution } & Objective \\
& & $x_{1}$ & $x_{2}$ & $x_{3}$ & function \\
\hline Model (26) & Algorithm 5 & 0.9996 & 0.0003 & 0.0001 & 0.4230 \\
Model (26) & Algorithm 6 & 0.6854 & 0.2304 & 0.0842 & 0.0425 \\
Model (26) & Algorithm 7 & 0.1562 & 0.6832 & 0.1606 & 0.0805 \\
\hline
\end{tabular}

$$
\begin{array}{ll}
\max & \operatorname{Ch}\left\{x_{1} k_{1}+x_{2} k_{2}+x_{3} k_{3} \geq 0.04\right\}(0.5) \\
\text { s.t. } & \sum_{i=1}^{3} x_{i}=1 \\
& x_{i} \geq 0 \quad i=1,2,3 .
\end{array}
$$

The parameters of Algorithm 5 are set up as follows.

(1) The parameter of birandom simulation is $N=2000$.

(2) The parameters of the improved LGMS-FOA are maxgen $=40$, sizepop $=30, r=40, d=20, w_{0}=1$, and $\rho=0.8$.

To show the efficiency of Algorithm 5, we choose two sets of parameters and the corresponding hybrid intelligent algorithms in $[7,8]$ which are named as Algorithms 6 and 7.

The parameters of Algorithm 6 are listed as below.

(1) The parameter of birandom simulation is $N=2000$.

(2) The parameters of GA are as follows: the iteration number is 40 , the population is 30 , the mutation probability is 0.6 , and the crossover probability is 0.1 .

The parameters of Algorithm 7 are given as follows.

(1) The parameter of birandom simulation is $N=2000$.

(2) The parameters of GA are as follows: the iteration number is 40 , the population is 30 , the mutation probability is 0.8 , and the crossover probability is 0.05 .

Remark 5. For Algorithms 5 to 7, the numbers of objective function computations are the same and this can ensure the fairness of comparisons.

5.2. Experimental Results. Compute models from (26) to (28) by using Algorithms from 5 to 7 , respectively, and repeat the experiments for 20 times. The average values of the optimal results are shown in Tables 2,3 , and 4 .

From Tables 2 to 4 , it can be found that the final searching quality of Algorithm 5 is better than Algorithms 6 and 7. So it can be concluded that the new hybrid intelligent algorithm is more efficient and precise than the existing algorithms when the numbers of objective function computations are the same.
TABLE 3: The optimal results of model (27).

\begin{tabular}{lccccc}
\hline \multirow{2}{*}{ Model } & \multirow{2}{*}{ Algorithm } & \multicolumn{3}{c}{ Optimal solution } & Objective \\
& & $x_{1}$ & $x_{2}$ & $x_{3}$ & function \\
\hline Model (27) & Algorithm 5 & 0.9832 & 0.0043 & 0.0125 & 0.0159 \\
Model (27) & Algorithm 6 & 0.6818 & 0.2948 & 0.0234 & 0.0071 \\
Model (27) & Algorithm 7 & 0.6057 & 0.2634 & 0.1309 & 0.0062 \\
\hline
\end{tabular}

TABLE 4: The optimal results of model (28).

\begin{tabular}{lccccc}
\hline \multirow{2}{*}{ Model } & \multirow{2}{*}{ Algorithm } & \multicolumn{3}{c}{ Optimal solution } & Objective \\
& & $x_{1}$ & $x_{2}$ & $x_{3}$ & function \\
\hline Model (28) & Algorithm 5 & 0.0005 & 0.9987 & 0.0008 & 0.5367 \\
Model (28) & Algorithm 6 & 0.0908 & 0.8339 & 0.0753 & 0.3382 \\
Model (28) & Algorithm 7 & 0.1221 & 0.8703 & 0.0076 & 0.4052 \\
\hline
\end{tabular}

\section{Conclusion}

To solve birandom portfolio selection problems better, this paper designs a new hybrid intelligent algorithm which integrates the improved LGMS-FOA algorithm and birandom simulation. Comparisons between the new hybrid intelligent algorithm and the existing algorithms show that the new hybrid intelligent algorithm is more effective and precise when the numbers of the objective function computations are the same.

\section{Conflict of Interests}

The authors declare that there is no conflict of interests regarding the publication of this paper.

\section{Acknowledgment}

This paper is supported by the National Natural Science Foundation of China (Grant no. 71232004).

\section{References}

[1] H. Markowitz, "Portfolio selection," The Journal of Finance, vol. 7, no. 1, pp. 77-91, 1952.

[2] B. Gray and B. Hollified, "When will mean-variance efficient portfolio be well diversified?” The Journal of Finance, vol. 47, no. 5, pp. 1785-1809, 1992.

[3] M. Linden, "A model for stock return distribution," International Journal of Finance \& Economics, vol. 6, no. 2, pp. 159-169, 2001.

[4] A. B. Romanovsky, "On version state recovery and adjudication in class diversity," Computer Systems Science and Engineering, vol. 17, no. 3, pp. 159-168, 2002.

[5] F. Jian-Qiang and W. Fu-Xin, "A research on return distribution function of chinese stock-market," Chinese Journal of Management Science, vol. 11, pp. 82-90, 2007.

[6] D. Da-Yong and J. Wei-Dong, "A subjective model of the distribution of returns and empirical analysis," Chinese Journal of Management Science, vol. 15, pp. 112-120, 2007. 
[7] L. Mei-Yan, "Chance-constrained portfolio selection with birandom returns," Modern Applied Science, vol. 3, no. 4, pp. 161165, 2009.

[8] L. Mei-Yan, "One type of optimal portfolio selection in birandom environments," Modern Applied Science, vol. 3, no. 6, pp. 121-126, 2009.

[9] G.-H. Cao and D. Shan, "The effect of exit strategy on optimal portfolio selection with birandom returns," Journal of Applied Mathematics, vol. 2013, Article ID 236579, 6 pages, 2013.

[10] J. Peng and B.-D. Liu, "Birandom variables and birandom programming," Computers and Industrial Engineering, vol. 53, no. 3, pp. 433-453, 2007.

[11] W. T. Pan, "A new fruit fly optimization algorithm: taking the financial distress model as an example," Knowledge-Based Systems, vol. 26, pp. 69-74, 2012.

[12] D. Shan, G.-H. Cao, and H.-J. Dong, "LGMS-FOA: an improved fruit fly optimization algorithm for solving optimization problems," Mathematical Problems in Engineering, vol. 2013, Article ID 108768, 9 pages, 2013.

[13] X. He, C. Li, T. Huang, C. J. Li, and J. Huang, "A recurrent neural network for solving bilevel linear programming problem," IEEE Transactions on Neural Networks and Learning Systems, vol. 25, no. 4, pp. 824-830, 2014.

[14] X. He, C. Li, T. Huang, and C. J. Li, "Neural network for solving convex quadratic bilevel programming," Neural Networks, vol. 51, pp. 17-25, 2014. 


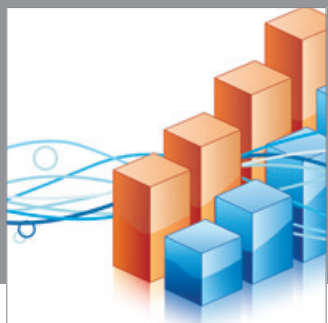

Advances in

Operations Research

mansans

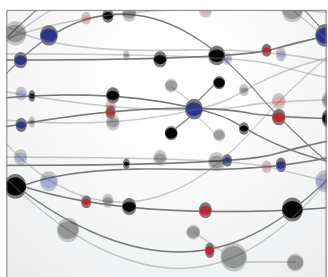

The Scientific World Journal
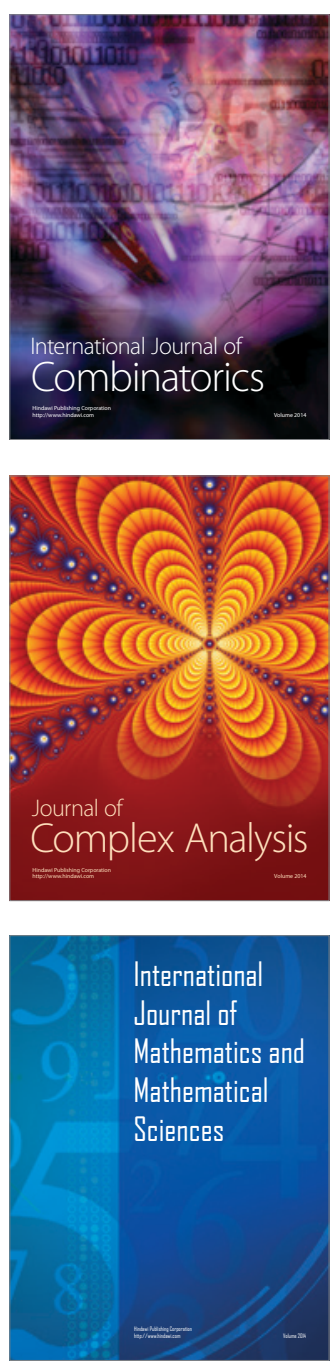
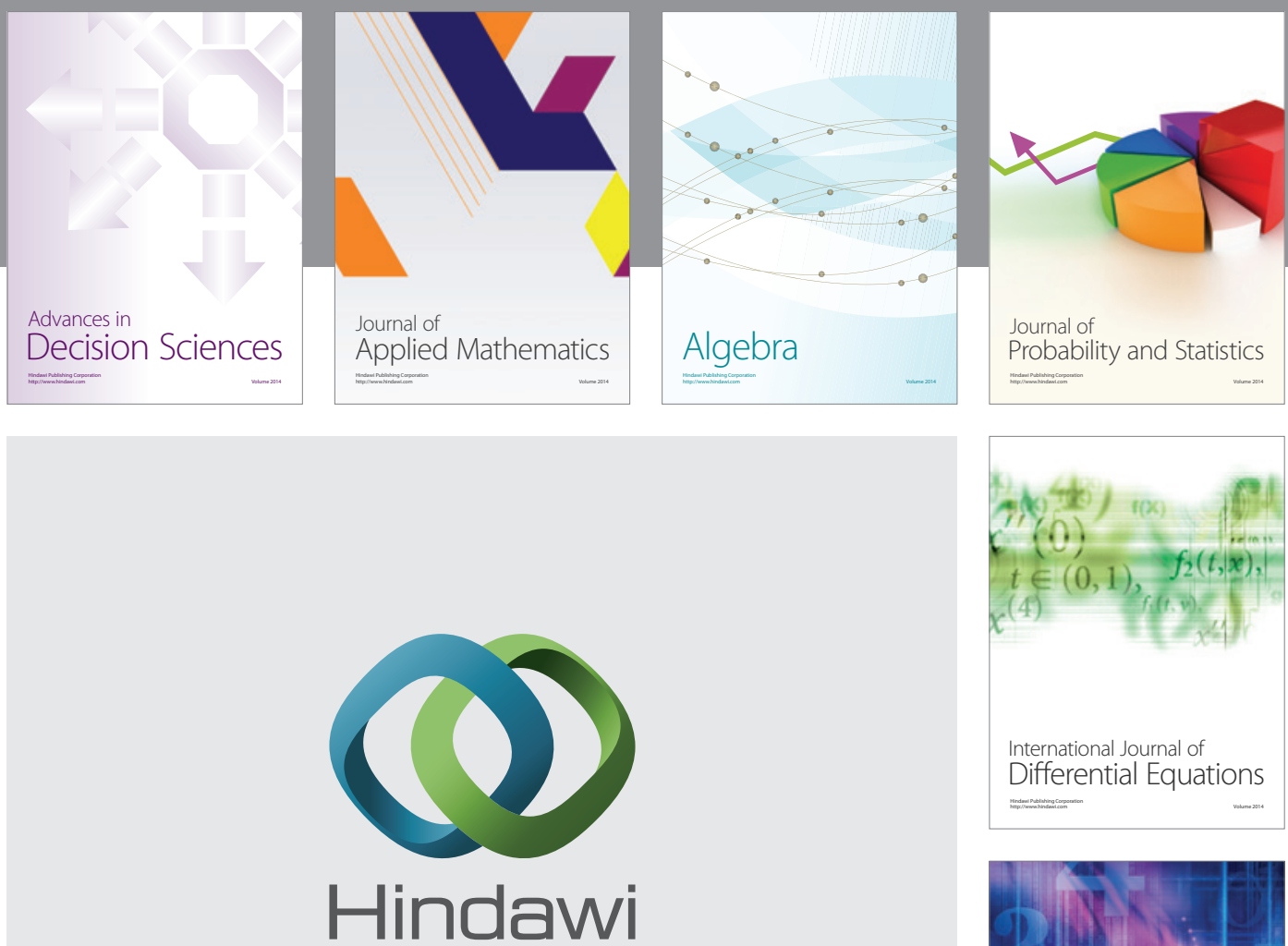

Submit your manuscripts at http://www.hindawi.com
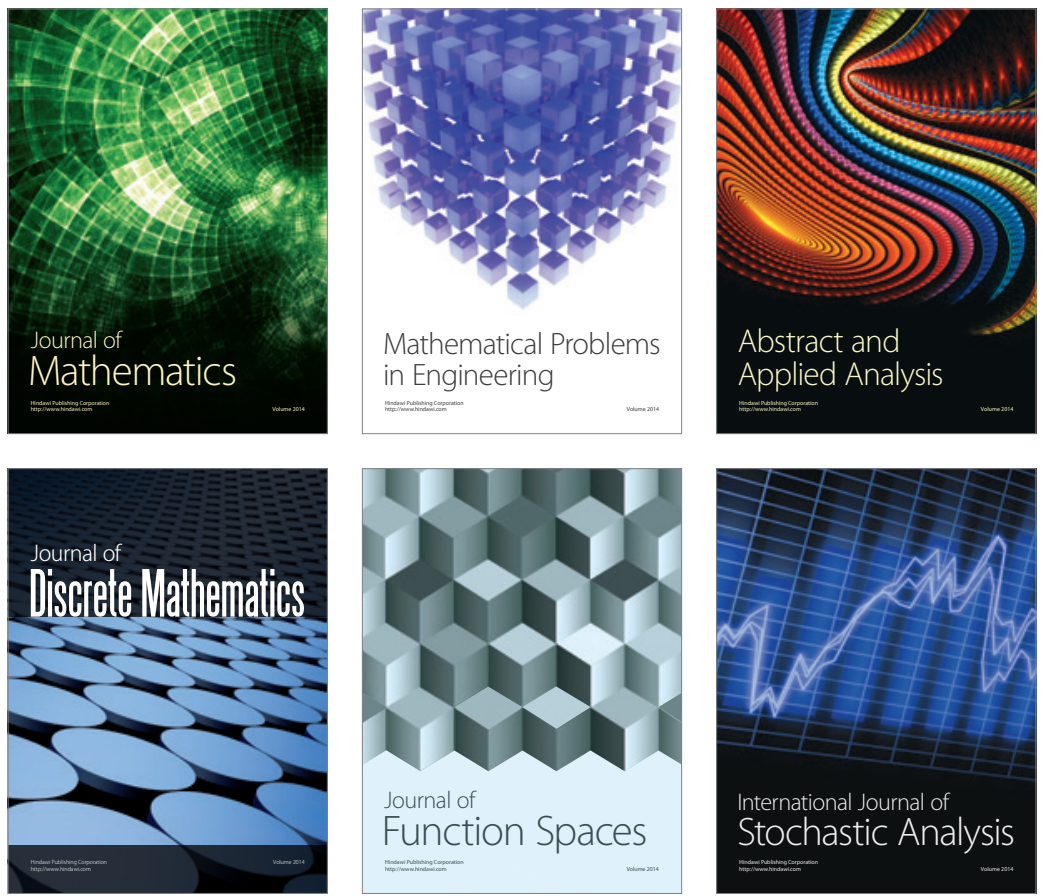

Journal of

Function Spaces

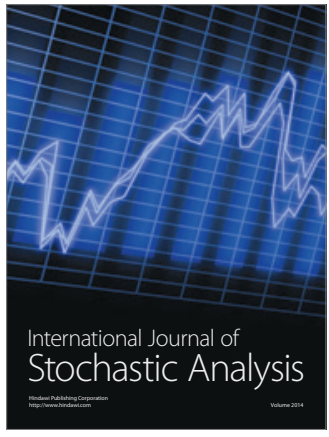

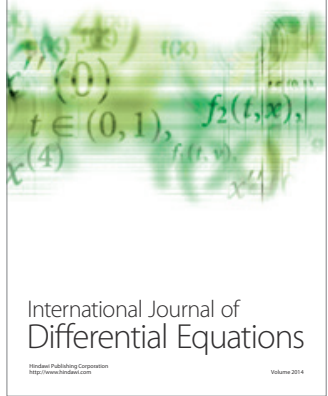
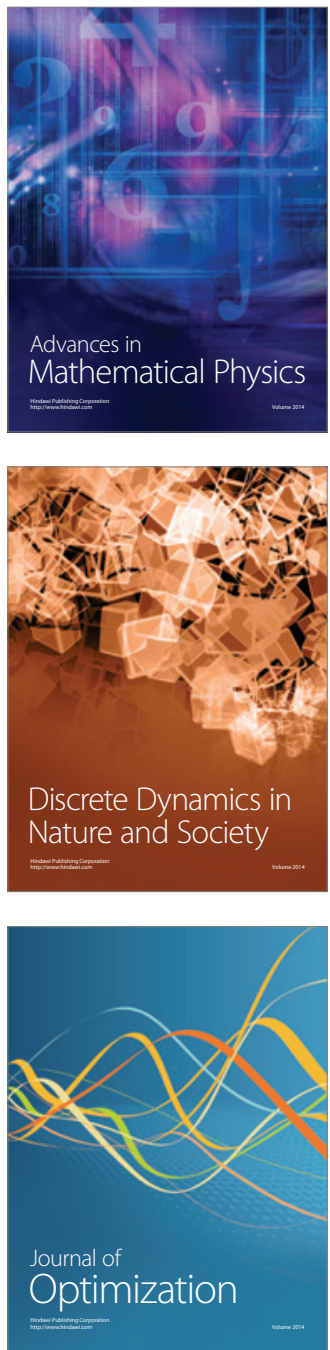